Штода М. Н.

\title{
ВЫБОР ГРАНИЧНЫХ УСЛОВИЙ ПРИ МОДЕЛИРОВАНИИ ПРОЦЕССА СОРТОВОЙ ПРОКАТКИ
}

Появление и распространение вычислительной техники в конце 60-х, начале 70-х гг. прошлого века коренным образом изменило развитие науки и техники. Начиная с этого времени, во всех областях научного знания появились разделы, так или иначе связанные с применением компьютерных технологий. Отдельно, но не маловажно, стоит область, получившая название - компьютерное (математическое) моделирование.

Теория обработки металлов давлением (ОМД), как прикладная наука, не является исключением, в этом смысле. Доказательством чего служит количество литературы, посвященной методам математического моделирования в ОМД и результатам исследований на основе компьютерного моделирования процессов ОМД, которое с каждым годом неуклонно возрастает. Такая тенденция легко объяснима, ведь «математическое моделирование процессов и решение задач обработки металлов давлением с применением численных методов существенно расширило возможности анализа и реализации новых энерго- и материалосберегающих технологий и их автоматического управления» [1, стр. 7].

Развитие современных методов компьютерного моделирования привело к тому, что в настоящее время параллельно и одинаково успешно используются как эмпирические, так и теоретические методы расчета деформации и напряженного состояния металла при прокатке в калибрах. Экспериментальные методы по определению являются упрощенными, так как чаще всего основываются на приведении сложной формы калибра и раската к прямоугольнику. Среди теоретических методов исследований наибольшее распространение получили методы, основанные на вариационных принципах механики деформируемого тела.

Успешность упрощенных подходов базируется на использовании многолетнего опыта эксплуатации прокатного оборудования и огромного количества лабораторных и промышленных исследований. В результате которых были разработаны методики использующих опытные коэффициенты, с труднообъяснимым физическим смыслом и, зависящие от всех основных факторов рассматриваемого процесса [2], но позволяющие получить удовлетворительную точность решения.

В то же время, применение вариационных принципов механики деформируемого тела позволяет разработать общие методы теоретического решения трехмерных задач ОМД, которые могут быть использованы для моделирования процессов обработки металлов давлением любой сложности, например, работы [3, 4, 5 и др.]. Однако, часто, точность теоретического решения таких задач не удовлетворяет конечного потребителя - производителей прокатной продукции и других видов обработки металлов давлением.

В результате такого положения вещей возник разрыв, и он, следует отметить, постоянно растет, между «практиками» и «теоретиками» прокатного производства. Одни, наблюдая за внешними изменениями процесса и не имея должного инструмента для глубокого его исследования, не могут с полной уверенностью объяснить причины, приведшие к этому изменению. Другие, имеющие неплохой математический аппарат, но по ряду причин все же не могут грамотно и обоснованно задать граничные условия, которые и так при моделировании заданы или учтены в расчетах с некоторыми упрощающими допущениями. В результате публикации одних лишены глубокого теоретического анализа и не могут дать ответ на физическую природу процесса, работы вторых могут привести к появлению ошибочного понимания изучаемого явления. По субъективному мнению автора, второе гораздо опаснее первого.

Особое внимание здесь следует уделить той части публикаций, в которых присутствуют результаты компьютерного моделирования и для доказательства достоверности 
результатов вместо эксперимента предложен авторитет используемого программного продукта. Судить о достоверности результатов таких работ трудно.

Из всего вышесказанного не следует, что необходимо отказаться от методов математического моделирования при исследовании процессов прокатки. Наоборот, автор считает, что аппарат компьютерного моделирования позволяет расширить возможности исследования столь сложного для изучения процесса. Однако, при этом не следует забывать, что мерилом достоверности все же является практика. И, поэтому, при проведении теоретических исследований адекватность расчетных моделей и заданных в них граничных условий следует проверять на практических результатах.

Целью данной работы является определение адекватного закона трения, описывающего контактные условия в очаге деформации при моделировании в программном продукте QForm 9.0.7 процесса прокатки свинцовых образцов в калибрах простой формы, так как при постановке задач процессов ОМД наибольшие трудности вызывают условия на контакте обрабатываемого металла с инструментом.

В настоящее время при моделировании процессов ОМД наибольшее распространение получили две теории распределения контактных сил трения:

- теория сухого трения, согласно которому прокатываемый металл скользит по всей дуге контакта (известный как закон Амонтона-Кулона) [6]:

$$
\tau_{\mathrm{x}}=\mathrm{f} \cdot \mathrm{p}_{\mathrm{x}},
$$

где $\tau_{\mathrm{x}}$ - касательное напряжение на контактной поверхности, вызванное силами трения между прокатываемым металлом и поверхностью валков;

f - коэффициент трения;

$\mathrm{p}_{\mathrm{x}}$ - нормальное напряжение на контактной поверхности (давление валков на прокатываемый металл).

- теория постоянных контактных сил трения (известная как закон Зибеля) [7]:

$$
\tau_{\mathrm{x}}=\mathrm{f} \cdot 2 \mathrm{k},
$$

где $\mathrm{k}$ - максимальное касательное напряжение при чистом сдвиге для деформируемого материала.

$$
\mathrm{k}=\frac{\sigma_{\mathrm{s}}}{\sqrt{3}}
$$

где $\sigma_{\mathrm{s}}$ - напряжение сопротивления деформации прокатываемого материала.

Кроме приведенных законов трения, программный продукт фирмы «КванторФорм» QForm 9.0.7 позволяет использовать 2 модификации смешанных законов трения: закон трения Леванова и смешанный закон трения, представляющий комбинацию законов трения Кулона и Зибеля.

Согласно закону трения Леванова А. Н. контактные силы трения в очаге деформации подчинены зависимости

$$
\tau_{\mathrm{x}}=\mathrm{m} \cdot \mathrm{k}\left(1-\mathrm{e}^{-\mathrm{b} \frac{\sigma_{\mathrm{n}}}{\sigma_{\mathrm{s}}}}\right)
$$

где $\mathrm{m}$ - фактор трения;

b - экспериментальный коэффициент (по рекомендации Леванова А.Н. $\mathrm{b}=\mathrm{b}_{0}=1,25$ );

$\sigma_{\mathrm{n}}-$ контактное нормальное напряжение.

Для получения практических данных, которые будут основанием определения адекватных контактных условий при моделировании, на лабораторном стане были прокатаны 
свинцовые образцы (свинец С1) круглого поперечного сечения со средним диаметром 6,62 мм в калибре с размерами, указанными на чертеже рис. 1. Прокатку производили на стане 210 с частотой вращения валков 100 об./мин. Для получения разных значений коэффициента трения на контакте раската с валками образцы перед прокаткой или обезжиривали, или покрывали мылом или мелом. Результаты измерений образцов до и после прокатки на лабораторном стане приведены в табл. 1.

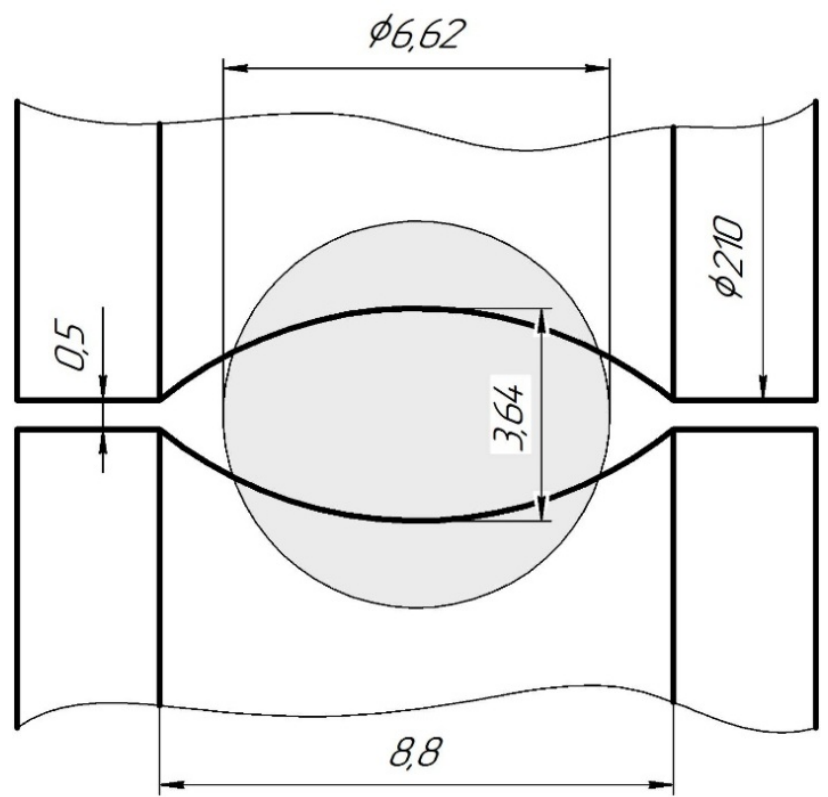

Рис. 1. Схема очага деформации во время исследований

Таблица 1

Результаты лабораторных исследований при разных контактных условиях ( $f=0,07$ - заготовка, покрытая мылом; $f=0,28$ - обезжиренная заготовка; $\mathrm{f}=0,43$ - заготовка, покрытая мелом)

\begin{tabular}{|c|c|c|c|c|c|c|}
\hline № & $\mathrm{H}_{0}$ & $\mathrm{~B}_{0}$ & $\mathrm{H}_{1}$ & $\overline{B_{1}}$ & \multirow{2}{*}{$\beta$} & \multirow{2}{*}{$\mathrm{f}$} \\
\hline & MM & MM & MM & MM & & \\
\hline 1 & 6,7 & 6,6 & 3,62 & 7,87 & 1,192 & \multirow{5}{*}{0,07} \\
\hline 2 & 6,68 & 6,59 & 3,64 & 7,9 & 1,199 & \\
\hline 3 & 6,66 & 6,6 & 3,63 & 7,86 & 1,191 & \\
\hline 4 & 6,67 & 6,65 & 3,71 & 7,86 & 1,182 & \\
\hline 5 & 6,65 & 6,67 & 3,74 & 7,91 & 1,186 & \\
\hline 6 & 6,72 & 6,66 & 3,72 & 9,72 & 1,459 & \multirow{5}{*}{0,28} \\
\hline 7 & 6,72 & 6,68 & 3,71 & 9,99 & 1,496 & \\
\hline 8 & 6,74 & 6,71 & 3,7 & 9,9 & 1,475 & \\
\hline 9 & 6,71 & 6,63 & 3,72 & 9,78 & 1,475 & \\
\hline 10 & 6,69 & 6,6 & 3,73 & 9,44 & 1,430 & \\
\hline 11 & 6,67 & 6,59 & 3,66 & 10,95 & 1,662 & \multirow{5}{*}{0,43} \\
\hline 12 & 6,66 & 6,59 & 3,7 & 10,88 & 1,651 & \\
\hline 13 & 6,66 & 6,61 & 3,73 & 10,85 & 1,641 & \\
\hline 14 & 6,66 & 6,62 & 3,75 & 10,82 & 1,634 & \\
\hline 15 & 6,69 & 6,62 & 3,75 & 10,89 & 1,645 & \\
\hline
\end{tabular}


По средним значениям, полученным при реальном процессе прокатки в лабораторных условиях, в программном продукте QForm 9.0.7 производили расчеты для разных коэффициентов трения. Для сокращения времени на решение задачи с учетом симметрии процесса для модели QForm были построены четверть калибра и четверть заготовки.

При создании операции были выбраны параметры типа операции - 3D деформация с учетом тепловых процессов. В качестве материала заготовки был выбран $\mathrm{Pb}$ cold из стандартной библиотеки, что близко соответствует натурному эксперименту. Температура прокатываемого материала $22{ }^{\circ} \mathrm{C}$. Параметры инструмента: создан универсальный привод с вращением инструмента вокруг оси против часовой стрелки с частотой 100 об./мин. Контактные условия варьировались: без смазки закон трения Кулона с коэффициентом трения 0,$13 ; 0,25 ; 0,4$ и 0,7; закон трения Зибеля с фактором трения 0,2; 0,5; 0,8 и 1,0; а также закон трения Леванова А. Н. с фактором трения 0,$5 ; 0,8$ и 1,0 и коэффициент $\mathrm{b}=1,25$. Сетка в заготовке имеет максимальный размер 0,8 мм. Остальные граничные условия выбраны по умолчанию.

Математическое моделирование в программе QForm позволяет визуализировать процесс прокатки поэтапно, при этом моделируются и переходные процессы момента захвата заготовки валками. Особенность реализации математической модели состоит в том, что часто самозахват полосы валками, в отличие от реального процесса, не происходит. Поэтому приходится идти на различные ухищрения. Например, создавать заталкивающий инструмент, который действует лишь в начальный момент процесса. Здесь же, при моделировании был применен другой прием: первые этапы расчета, до полного заполнения металлом очага деформации (см. рис. 2), производили с повышенным коэффициентом трения по закону Кулона 0,8. Это обеспечило надежный захват раската валками. Далее изменяли условия на контакте на необходимый закон трения с определенным коэффициентом (фактором) трения и продолжали расчет после текущего шага с новыми граничными условиями.

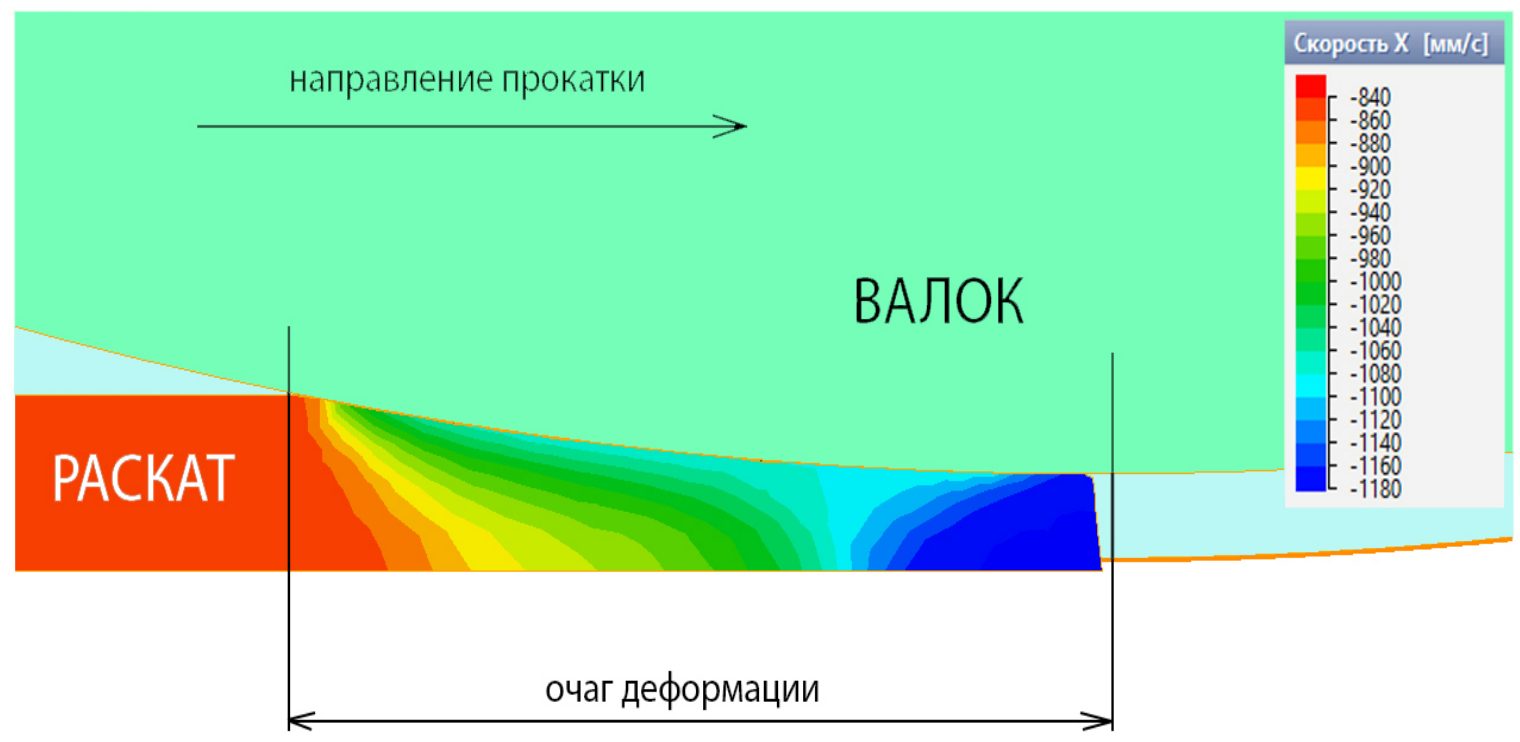

Рис. 2. Поле продольных скоростей $\mathrm{v}_{\mathrm{x}}$ в вертикальной плоскости симметрии. Результаты расчета в программе QForm после 44 шага

По окончании расчетов с разными условиями на контакте графически сопоставили значения коэффициента уширения по экспериментальным и теоретическим данным (рис. 3).

Как видно из рис. 3, характер влияния коэффициента трения на величину коэффициента уширения при прокатке круглых свинцовых полос в овальных калибрах, полученный на основании результатов расчетов в программном продукте QForm 9.0.7 и в результате лабораторных исследований совпадает: с увеличением коэффициента трения коэффициент уширения возрастает. Это объясняется тем, что при прокатке узких полос $\left(\frac{\mathrm{b}_{\mathrm{cp}}}{\mathrm{R}_{\mathrm{K}} \alpha_{\mathrm{cp}}}<1\right)$ и при 
прокатке в калибрах, увеличение коэффициента трения приводит к более интенсивному возрастанию продольных подпирающих сил, чем поперечных. Поэтому с ростом сил трения в очаге деформации, в рассматриваемых случаях, возрастает уширение.

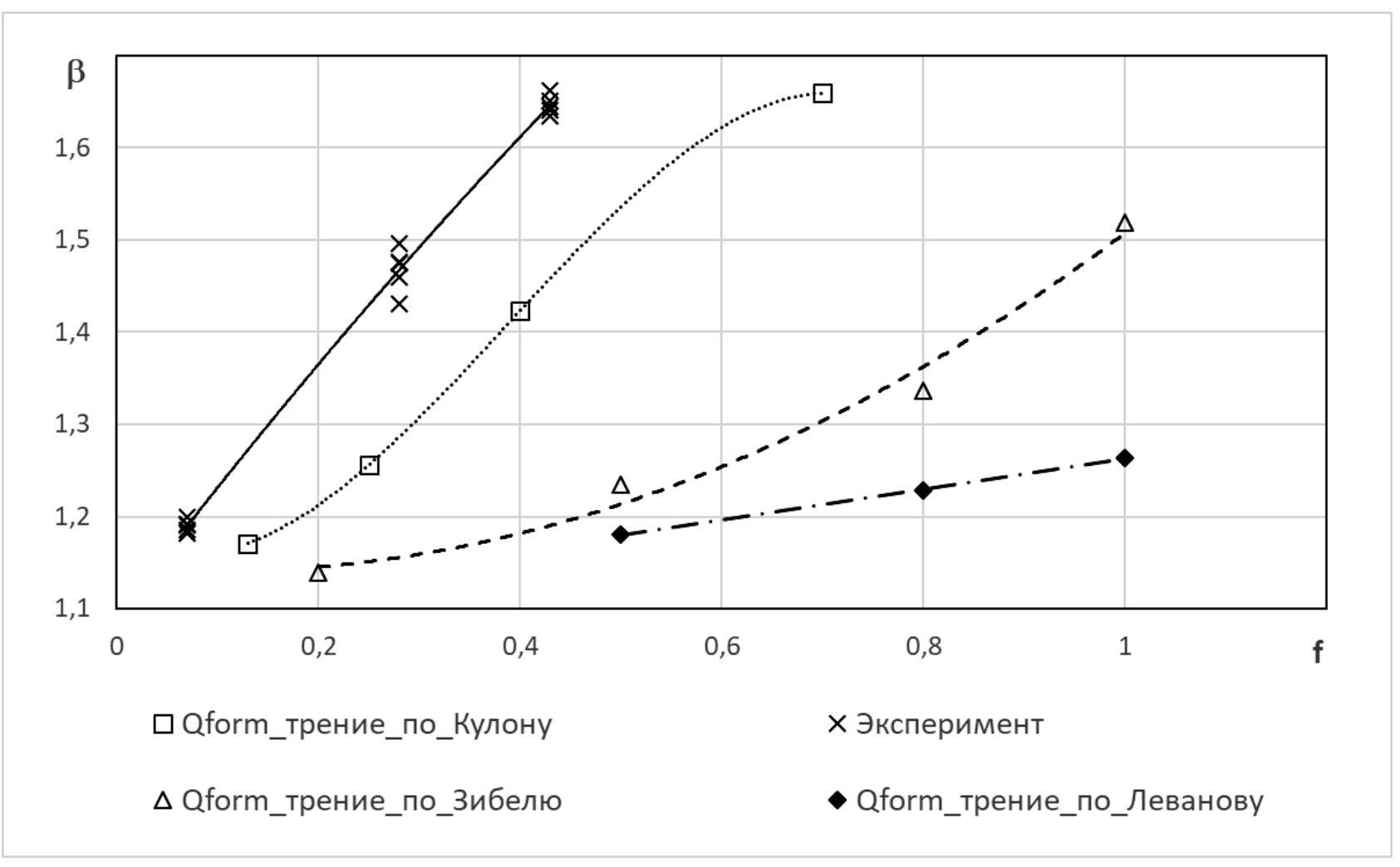

Рис. 3. Теоретическая и экспериментальная зависимости коэффициента уширения от коэффициента трения на контакте металла с валками

Из рис. 3 видно, что наиболее близкие к экспериментальным данным были результаты расчетов с применением закона трения Кулона. Законы трения Зибеля и Леванова А. Н. для моделирования процесса прокатки в калибрах применять нельзя. Для проведенных в работе условий лабораторного эксперимента соответствуют следующие условия трения по закону Кулона при моделировании в программном продукте QForm: для заготовок, покрытых мылом $-\mathrm{f}=0,15$ (реальный коэффициент трения $\mathrm{f}=0,07$ ); для заготовок, покрытых мелом $\mathrm{f}=0,6$ (реальный коэффициент трения $\mathrm{f}=0,43$ ); а для сухих (обезжиренных) свинцовых образцов $-\mathrm{f}=0,45$ (реальный коэффициент трения $\mathrm{f}=0,28)$.

\section{ВЫВОДЫ}

В статье показана необходимость и эффективность проведения инверсного анализа для определения граничных условий при моделировании процессов ОМД.

Показано, что все законы трения, предлагаемые в программном продукте QForm, адекватно описывают характер влияния коэффициента трения на распределение течения металла в продольном и поперечном направлениях. Наибольшая точность расчетов получена при использовании закона трения Кулона, который рекомендовано использовать при моделировании процессов прокатки в программном продукте QForm. Законы трения Зибеля и Леванова А. Н. для моделирования процесса прокатки в калибрах применять нельзя.

В статье показано, что для данных условий лабораторного эксперимента соответствуют следующие условия трения по закону Кулона при моделировании в программном продукте QForm: для заготовок, покрытых мылом - f = 0,15 (реальный коэффициент трения 
$\mathrm{f}=0,07)$; для заготовок, покрытых мелом $-\mathrm{f}=0,6$ (реальный коэффициент трения $\mathrm{f}=0,43$ ); а для сухих (обезжиренных) свинцовых образцов $-\mathrm{f}=0,45$ (реальный коэффициент трения $\mathrm{f}=0,28)$.

\section{СПИСОК ИСПОЛЬЗОВАННОЙ ЛИТЕРАТУРЫ}

1. Данченко В. Н., Миленин А. А., Кузьменков В. И., Гринкевич В. А. Компьютерное моделирование процессов обработки металлов давлением. Численные методы. Днепропетровск: «Системные технологии», 2005. 448 с.

2. Тарновский И. Я., Скороходов А. Н., Илюкович Б. М. Элементы теории прокатки сложных профилей. Москва: Металлургия, 1972. 352 с.

3. Алиева Л. И. Оценка деформированного состояния деталей в процессе холодного выдавливания. Металлургическая и горнорудная промышленность. 2016. 2. С. 77-85.

4. Ремез О. А., Огинский И. К., Кузьмина О. М. Моделирование процесса непрерывной прокатки в калибрах системы «овал-круг». Обработка материалов давлением. Краматорск: ДГМА. 2019. 1 (48). С. 63-70.

5. Смирнов Е. Н., Скляр В. А., Уразова Л. Ф., Пивоваров Р. Е. Математическое моделирование поведения дефектов макроструктуры непрерывнолитого металла в процессе прокатки. Вісник HTУ «XПI». Cерія «Iнноваційні технологї та обладнання обробки матеріалів у машинобудуванні та металургї̈. 2016.38 (1210). C. 41-47.

6. Грудев А. П. Внешнее трение при прокатке. Москва: Металлургия, 1973. 288 с.

7. Целиков А. И. Основы теории прокатки. Москва: Металлургия, 1965. 248 с.

\section{REFERENCES}

1. Danchenko V.N., Milenin A.A., Kuzmenkov V.I., Grinkevich V.A. Computer modeling of metal forming processes. Numerical methods. Dnepropetrovsk: System technologies. 2005. 448 p. (in Russian).

2. Tarnovsky I.Ya., Skorokhodov A.N., Ilyukovich B.M. Elements of the theory of rolling complex profiles. Moscow: Metallurgy. 1972. 352 p. (in Russian).

3. Aliieva L.I. Assessment of the deformed state of parts in the process of cold extrusion. Metallurgical and mining industry. 2016. 2, pp. 77-85. (in Russian).

4. Remez O.A., Oginsky I.K., Kuzmina O.M. Modeling the process of continuous rolling in the calibers of the “oval-circle” system. Materials Working by Pressure. Kramatorsk: DSEA. 2019. 1 (48), pp. 63-70. (in Russian).

5. Smyrnov Y.N., Skliar V.A., Urazova L.F., Pivovarov R.Y. Mathematical modeling of the behavior of defects macrostructure of the continuous cast metal in the rolling process. Bulletin of NTU "KhPI". Series: Innovative technologies and equipment handling materials in mechanical engineering and metallurgy. 2016.38 (1210), pp. $41-47$. (in Russian).

6. Grudev A.P. External rolling friction. Moscow: Metallurgy, 1973. 288 p. (in Russian).

7. Tselikov A.I. Foundations of rolling theory. Moscow: Metallurgy, 1965. 248 p. (in Russian).

Штода М. Н. - канд. техн. наук, доц. ДГТУ.

ДГТУ - Днепровский государственный технический университет, г. Каменское.

E-mail: maksshtoda@gmail.com; https://orcid.org/0000-0002-3918-2492 